Relations industrielles

Industrial Relations

\title{
Introduction
}

\section{Gregor Murray}

Volume 57, numéro 1, hiver 2002

URI : https://id.erudit.org/iderudit/006708ar

DOI : https://doi.org/10.7202/006708ar

Aller au sommaire du numéro

Éditeur(s)

Département des relations industrielles de l’Université Laval

ISSN

0034-379X (imprimé)

1703-8138 (numérique)

Découvrir la revue

Citer cet article

Murray, G. (2002). Introduction. Relations industrielles / Industrial Relations, 57(1), 8-11. https://doi.org/10.7202/006708ar

Tous droits réservés @ Département des relations industrielles de l’Universite Laval, 2002
Ce document est protégé par la loi sur le droit d'auteur. L'utilisation des services d'Érudit (y compris la reproduction) est assujettie à sa politique d'utilisation que vous pouvez consulter en ligne.

https://apropos.erudit.org/fr/usagers/politique-dutilisation/ 


\section{Introduction}

L'entreprise transnationale devient de plus en plus le porte-étendard des forces de la mondialisation. En produisant au moins $25 \%$ du produit intérieur brut du globe et en comptant pour autant que les deux-tiers du commerce mondial, en employant au moins 75 millions de travailleurs (dont plus de la moitié vit à l'extérieur de leur pays d'origine) et en étant de plus en plus intégrée à des réseaux de production complexes et globaux, l'entreprise transnationale, dont la croissance spectaculaire et les tentacules s'étendent à tous les coins de la planète, soulève des questions clefs dans les domaines de l'organisation du travail et de la relation d'emploi en général.

C'est particulièrement le cas en matière de législation du travail. Les lois nationales du travail doivent composer avec une tension fondamentale entre des réseaux globaux de production qui transcendent les frontières d'un pays et le fait que les succursales des entreprises transnationales soient intégrées à des territoires nationaux et soient assujetties aux lois de ces juridictions locales. Savoir comment atteindre les niveaux réels de prise de décision d'une entreprise devient alors une préoccupation particulière, a fortiori lorsque des décisions prises dans une partie du monde ont un impact sur les relations d'emploi dans des juridictions tout à fait différentes ailleurs dans le monde. Même si des décisions au plan des relations d'emploi peuvent être parfois «locales » de par leur nature, elles sont souvent transnationales. En empruntant des contributions traitant de ce même thème au Colloque de novembre 2000 organisé par la Société québécoise de droit du travail et de la sécurité sociale, dont nous devons remercier notre éditeur de $R I / I R$, Pierre Verge, pour son inspiration, ce numéro de $R I / I R$ réunit deux tentatives importantes de systématisation de nos connaissances sur ce sujet.

Dans un premier temps, Pierre Verge et Sophie Dufour centrent leur attention sur ce «dualisme contradictoire » entre des frontières nationales et des réalités internationales en vue de donner un aperçu de l'état actuel de la législation du travail eu égard à l'entreprise transnationale et d'en donner une appréciation. Même si le droit national manifeste toujours une incapacité à saisir ce caractère transfrontalier de l'entreprise transnationale, ils identifient certains domaines où la législation du travail arrive dans au moins une certaine mesure à transcender les frontières d'un pays et à encadrer les activités des entreprises transnationales. 
Dans un second temps, étant donné la nature inégalitaire du développement économique qui caractérise la politique économique internationale, la plupart des entreprises transnationales ont leurs sièges sociaux dans un nombre limité de pays ; il en est ainsi surtout aux États-Unis. Cette concentration des transnationales et la permissivité relative des cours américaines à l'endroit des recours collectifs incitent Lance Compa à évaluer à l'aide de la jurisprudence récente l'émergence d'une promotion des droits internationaux du travail dans les cours de justice aux États-Unis. En dépit des nombreux obstacles à la poursuite de tels recours dont on fait état dans cet article, Compa fait l'argumentaire pour ce type de stratégie judiciaire comme étant un moyen parmi plusieurs qui peut s'avérer efficace dans la promotion des droits des travailleurs dans l'économie mondiale.

Ces deux articles dans leur ensemble mettent en évidence la fluidité actuelle du cadre de référence de la législation ouvrière et de son évolution probable vers un accommodement avec l'entreprise transnationale. Ils présentent également une démonstration convaincante de l'importance de la recherche qui s'intéresse à la fois au national et au transnational au plan de la conceptualisation et de l'analyse.

Le troisième article de ce numéro, celui de Philippe Bernoux, un des leaders en France en théorie des organisations, est une réponse à un défi d'ordre théorique important : chercher à expliquer le changement dans les organisations. À une époque où la gestion du changement devient le leitmotiv en matière de survie organisationnelle, Bernoux soutient qu'une approche interactionniste est la seule qui puisse fournir un cadre adéquat d'analyse à la compréhension du changement. En s'appuyant à la fois sur la recherche empirique actuelle et une littérature théorique plus vaste, il tente de démontrer que l'innovation n'est ni le produit de facteurs externes ni inévitablement le résultat de nouveaux systèmes de gestion. L'innovation s'installe plutôt par le biais de l'interaction sociale quotidienne sur les lieux de travail, en prenant comme assises le consensus chez les travailleurs et les modes de régulation autonome. De ces facteurs découle la performance d'une entreprise et c'est ainsi que le changement peut trouver une explication.

Dans un quatrième article, Joseph Rose présente un cas type saisissant d'efforts de réorganisation des dépenses publiques : celui de la négociation chez les enseignants de l'élémentaire et du secondaire en Ontario au cours de la dernière décennie. Il se demande si les changements dans la structure et la pratique de la négociation collective dans ce secteur répondent à une logique de coûts, d'idéologie ou de problèmes liés à l'efficacité du système particulier de négociation collective. En analysant cette expérience, Rose en vient à la conclusion que l'idéologie du gouvernement et les considérations de coûts sont à la racine de la recherche d'un changement. Dans 
un contexte où les gouvernements de d'autres provinces au Canada et ailleurs cherchent à réduire les coûts dans le secteur de l'éducation, l'auteur met également en évidence la multitude des conséquences tant prévues qu'inattendues de ces changements en Ontario, des conséquences qui devraient inciter à la prudence avant d'amorcer ailleurs de telles réformes dans ce secteur.

De l'analyse comparative entre le Canada et les États-Unis de la croissance ou du déclin du taux de syndicalisation, deux idées ont monnaie courante : à savoir une préoccupation à l'endroit d'un ensemble de lois ouvrières qui seraient plus favorables au Canada et la présence de valeurs sociales différentes venant susciter chez les employeurs une opposition moins féroce à la syndicalisation au Canada. Les deux articles qui viennent clore ce numéro de RI/IR nous invitent à revoir ces idées reçues.

D'abord, tout en cherchant à démontrer une évolution vers des lois du travail plus favorables, John Logan met à jour un paradoxe historique remarquable. Pendant plusieurs décennies, suite à la consolidation du régime moderne d'après-guerre des relations industrielles au Canada, les lois du travail canadiennes étaient considérées comme moins propices à la syndicalisation que l'étaient les lois américaines. Cependant, c'était le caractère plus restrictif de la législation canadienne, même si elle était initialement plus sévère à l'endroit de l'accréditation syndicale et les mouvements de grève, qui offrait apparemment moins d'espace à l'opposition des employeurs. En s'appuyant sur sa recherche historique, Logan retrace les positions des employeurs à l'égard du cadre légal canadien, leur opposition constante à un cadre légal plus favorable aux syndicats et à des modifications récentes susceptibles d'être plus propices à la syndicalisation.

En second lieu, dans un apport empirique important à ce débat, Karen Bentham analyse l'existence d'une opposition des employeurs à la syndicalisation au Canada. Une conclusion significative met en évidence la profondeur et l'ampleur de l'opposition des employeurs à la syndicalisation. De plus, en cherchant à préciser l'influence de différentes formes d'opposition de la part des employeurs au cours de nombreuses « rondes » de négociation, l'auteur démontre que non seulement l'opposition des employeurs crée un impact sur la possibilité initiale d'obtenir une accréditation, mais encore qu'en longue période une telle résistance exerce une influence sur la création d'une relation de négociation stable.

Enfin, c'est avec une grande tristesse que nous avons appris le décès en janvier 2002 du président du comité de rédaction de la Revue $R I / I R$, le professeur Noah Meltz, qui fut pendant longtemps associé au Centre des relations industrielles de l'Université de Toronto. En plus de son apport remarquable à la communauté des relations industrielles au Canada, Noah fut longtemps un grand ami de RI/IR. Ses contributions nombreuses à la 
revue vont grandement nous manquer. Nous offrons aussi nos condoléances bien senties à sa famille, à ses amis et à ses collègues pour la perte de ce professeur et académicien des plus généreux.

Gregor Murray Directeur 